\title{
Real-time confidence of clinical decision making: a systematic review
}

\author{
Authors: Myura Nagendran ${ }^{A}$ and Yang Chen ${ }^{B}$
}

\section{Introduction}

Good decision making is at the core of providing high-quality healthcare to patients. Of the many cognitive biases that can warp decision making, under- or overconfidence are particularly insidious threats, given their universality and ability to anchor or prime individuals to choosing erroneous management strategies. ${ }^{1}$ A recent opinion piece highlighted the broader point that confidence does not always equal competence and this has also been reported in a survey of junior doctors. ${ }^{2,3}$ Given the difficulty of standardising experiments in this field, most studies tend to administer questionnaires that suffer from recall bias or assess confidence in structured vignettes away from the clinical shop floor. We sought to establish to what extent decision confidence has been measured in real time and whether confidence or lack thereof correlates with important outcomes.

\section{Methods}

We searched Medline, Embase and PsycINFO with synonyms for clinical decision making, meta-cognition and physicians on 1 February 2019. Two reviewers independently selected studies for inclusion if they assessed the confidence of clinical decision making (eg diagnosis or treatment decisions) by healthcare practitioners in real time. Vignette studies and surveys were excluded.

\section{Results and discussion}

The search retrieved 2,288 studies of which 10 initially seemed includable. Four studies and one conference abstract remained after full-text review. The Acute Abdominal Pain (AAP) study group prospectively assessed trainee and senior surgeon confidence at the point of diagnosing abdominal pain in the emergency department. ${ }^{4}$ Diagnostic accuracy between the two groups was comparable at $44 \%$ and $43 \%$ respectively, but trainees were less confident than seniors (seven versus eight out of 10). Boots et al found that diagnostic confidence for pneumonia was higher with more classical presentations or when pneumonia was considered 'life-threatening'.

Bruyninckx et al assessed general practitioner (GP) referrals for chest pain and reported an odds ratio (OR) for referral of 11.6 when GP diagnosis was certain versus 3.0 when uncertain. ${ }^{6}$ Davis and colleagues investigated confidence in emergency department

Authors: ${ }^{\mathrm{A}}$ Imperial College London; ${ }^{\mathrm{B}}$ University College London ultrasound-guided diagnosis and found that accuracy, sensitivity and specificity were $6 \%, 7 \%$ and $4 \%$ higher when confidence scores were 9 or $10 .{ }^{7}$ Nguyen et al asked physicians to predict the likelihood of readmission for patients on the day of discharge along with their confidence in the prediction. Physicians were more confident when they deemed readmission to be high risk versus low risk. ${ }^{8}$

\section{Conclusion}

The literature on real-time assessment of decision confidence is notably sparse and existing studies are small. Confidence among both trainees and seniors was surprisingly high in one study, despite only moderate diagnostic accuracy. Physician confidence in the primary care setting may have an impact on referral patterns. Further research in this area should establish the associations between decision confidence and other physician and patient factors in a larger sample, with a view to generating hypotheses for testable interventions that can better calibrate confidence with clinical decision making.

\section{References}

1 Croskerry P, Singhal G, Mamede S. Cognitive debiasing 1: origins of bias and theory of debiasing. BMJ Qual Saf 2013;22(Suppl 2):ii58-64.

2 Treadway NA. A student reflection on doctoring with confidence: mind the gap. Ann Intern Med 2018;169:564-5.

3 Barnsley L, Lyon PM, Ralston S] et al. Clinical skills in junior medical officers: a comparison of self-reported confidence and observed competence. Med Educ 2004;38:358-67.

4 Acute Abdominal Pain (AAP) Study group. Diagnostic accuracy of surgeons and trainees in assessment of patients with acute abdominal pain. Br J Surg 2016;103:1343-9.

5 Boots RJ, Lipman J, Bellomo R, Stephens D, Heller RE. Predictors of physician confidence to diagnose pneumonia and determine illness severity in ventilated patients. Australian and New Zealand practice in intensive care (ANZPIC II). Anaesth Intensive Care 2005;33:112-19.

6 Bruyninckx R, Van den Bruel A, Aertgeerts B, Van Casteren V, Buntinx $F$. Why does the general practitioner refer patients with chest pain not-urgently to the specialist or urgently to the emergency department? Influence of the certainty of the initial diagnosis. Acta Cardiol 2009;64:259-65.

7 Davis DP, Campbell C], Poste JC, Ma G. The association between operator confidence and accuracy of ultrasonography performed by novice emergency physicians. J Emerg Med 2005;29:259-64.

8 Nguyen OK, Patel V, Washington C, Makam AN. Variation in physician perceptions of readmission risk by physician specialty and perceived reason for readmission. J Gen Intern Med 2018;33(Suppl 2):S388. 